\title{
Design and Deployment of Aqua Monitoring System Using Wireless Sensor Networks and IAR-Kick
}

\author{
Suresh Babu Chandanapalli*, Sreenivasa Reddy $E^{2}$ and Rajya Lakshmi D ${ }^{3}$ \\ ${ }^{1}$ Associate Professor, Department of CSE, Gudlavalleru Engineering College, Gudlavalleru-521356, Andhrapradesh, India \\ ${ }^{2}$ Professor of CSE, ANU College of Engineering and Technology, Acharya Nagarjuna University, Guntur-522510, Andhrapradesh, India \\ ${ }^{3}$ Professor, HOD of CSE, JNTUK University College of Engineering, Vizianagaram-535002, Andhrapradesh, India
}

\begin{abstract}
In Aquaculture, the yields (shrimp, fish etc.) depend on the water characteristics of the aquaculture pond. For maximizing fish yields, the parameters which are to be kept at certain optimal levels in water are dissolved oxygen, temperature, salinity, turbidity, $\mathrm{pH}$ level, alkalinity and hardness, ammonia and nutrient levels. These parameters can vary a lot during the period of a day and can rapidly change depending on the external environmental conditions. Hence it is necessary to monitor these parameters with high frequency, if not continuously, for timely analysis and action. This need accurate real- time information system and performance in order to maximize their potential. Wireless sensor networks are used to monitor aqua farms for relevant parameters, such as $\mathrm{pH}$ levels, humidity, dissolved oxygen levels, water temperature, ammonia levels etc. This system consists of two modules which are transmitter station and receiver station. The transmitter station consists of sensor nodes such as $\mathrm{pH}$, humidity, and temperature inside and outside of water, and also microcontrollers, GSM, analog/digital converters. The receiver station consists of GSM module for receiving the sensing data from transmitter through GSM network. The receiver station receives data through the com port and stores in PC in order to achieve human-computer interface. The graphical user interface was designed, so that farmers and investigators can observe, investigate and analyze the related data. The user interface allows us to convey the analyzed data in the form of a message to the farmers in their respective local languages to their Mobile Phones and alerts them in unhygienic environmental conditions. With this even semi-literate farmers can interact with the system and can understand the information in order to take suitable actions.
\end{abstract}

Keywords: Aquaculture; Wireless sensor networks; IAR-Kick; PH

\section{Introduction}

Aquaculture has been a fast-growing industry because of significant increases in demand for fish and seafood throughout the world. Its economic importance is increasing economically. Aquaculture -- also known as fish or shellfish farming -- refers to the breeding, rearing, and harvesting of plants and animals in all types of water environments including ponds, rivers, lakes, and the ocean. Researchers and aquaculture producers are "farming" all kinds of freshwater and marine species of fish, shellfish, and plants. Aquaculture produces food fish, sport fish, bait fish, ornamental fish, crustaceans, mollusks, algae, sea vegetables, and fish eggs. Aquaculture consist Two types, one is Marine aquaculture refers to the culturing of species that live in the ocean. Another one is Freshwater aquaculture produces species that are native to rivers, lakes, and streams [1].

In shrimp culture, monitoring of ecological parameters allows the direct and smooth management of water quality in the ponds, so avoiding the incidence of unfavorable conditions that can be harmful for organisms [2]. Besides, some samples are observed to be most sensitive to low dissolved oxygen in water, temperature, salinity and $\mathrm{pH}$ level of the water. Taking into consideration of deploying sensors for monitoring water quality and also for giving early warnings regarding the contaminants in the water, the shrimp culture may yield better results [3].

Yet, one of the relevant continual issues faced by aquaculture farmers for efficiently monitoring the water quality of their fish ponds is the capability to monitor and adjust too many operations independently and simultaneously. The advances in sensor technologies, mobile computing, and wireless communications now allow water quality scientists to acquire, process and transmit an collection of data while still in the field or remotely from off-side laboratories. Real-time remote monitoring technologies offer several advantages over historical Observing systems by streamlining the information accumulation process, conceivably minimizing human lapses and time delays, considerably increasing the quantity and quality of data on temporal and spatial scales [4].

Recent analysis of water quality requires a constant observing of the distinctive water quality parameters in the significant catchments. This makes another standard in water quality sensing as the data is to be gathered in the end transferred wirelessly over a certain period of time. Accurate measurement of water quality requires measurement of parameters like $\mathrm{pH}$, dissolved oxygen, water temperature, water level, among others, at different depths on the high spatial determination. Measuring instruments should belong to wireless distributed sensor networks, small, and cheap sensors which would likely be the most ideal choice [5].

The pond production is limited by the availability of dissolved oxygen. Dissolved oxygen is the most discriminating quality parameter, since shrimps in the low dissolved oxygen are more vulnerable to

*Corresponding author: Suresh Babu Chandanapalli, Associate Professor Department of CSE, Gudlavalleru Engineering College, Gudlavalleru-521356, Andhrapradesh, India, Tel: +919676021214; E-mail: sureshdani2004@gmail.com

Received October 21, 2014; Accepted October 31, 2014; Published November 03, 2014

Citation: Chandanapalli SB, Sreenivasa Reddy E, Rajya Lakshmi D (2014) Design and Deployment of Aqua Monitoring System Using Wireless Sensor Networks and IAR-Kick. J Aquac Res Development 5: 283. doi:10.4172/2155-9546.1000283

Copyright: () 2014 Chandanapalli SB, et al. This is an open-access article distributed under the terms of the Creative Commons Attribution License, which permits unrestricted use, distribution, and reproduction in any medium, provided the original author and source are credited. 
diseases. Dissolved oxygen is required in various ways; it is required for fish breath, waste decomposition and algal respiration [6].

It is of extraordinary essentialness to all aquatic organisms as they reflect the physical and biological processes occurring in the water body. Besides, it is significant to the production and support of life as it is also necessary for the decomposition of organic matter.

Dissolved oxygen minimum levels are recommended to oscillate between 4 and $5 \mathrm{ppm}$ and is also suggested that the dissolved oxygen level should be kept above $2 \mathrm{ppm}$. Mineralization of organic matter and nitrification require oxygen and response rates require maintenance of oxygen at a rate above a critical threshold (typically $>2-3 \mathrm{mg} \mathrm{l}^{-1}$ ). The minimum oxygen threshold levels needed for nitrification is similar to the oxygen levels needed for god fish development [7].

Temperature also determines tropical shrimp growth and immune response. The demand of dissolved oxygen increases in high values of temperature, which stress shrimp resulting in high mortality rates. Temperature also controls toxicity of ammonia, chemical reactions and solubility of gasses [8].

Reduction of dissolved oxygen occurs due to high salinity concentrations. Stress on shrimp occurs due to extremely low or high $\mathrm{pH}$ which result in poor survival and soft shell water body with 6.5 to $9 \mathrm{pH}$ concentrations are appropriate and reproduction decreases alkaline death appears in values above 11 and acid death with values below than 4 [9].

These problems were recognized in recent years by regulatory agencies. They are tasked with evaluating water resources in attempts to ensure that environmental levels of physical variables are maintained within established compliance conditions. It resulted in programs initiated towards continuous surveying by onsite water monitoring instruments and constant monitoring is carried out to detect changes and trends in critical parameters. Continuous environmental parameter monitoring provides an important early warning information to decision-makers [4].

Real-time information systems developed and deployed at lowcost short-range modules of wireless sensor network are based on Zig Bee standard and virtual instrument technology to monitor and control an aquaculture system. The system consists of smart sensor nodes, coordinator/gateway node and personal computer (PC). The smart sensor nodes monitor ecological parameters such as, water level, water temperature, and humidity $\mathrm{pH}$ and dissolved oxygen using relevant sensors and send this information to the coordinator/ gateway node through ZigBee network and receive control signals for actuator control. The coordinator/gateway node receives data acquired and sends command to PC in order to make human-computer visualization interface.

Wireless sensor networks (WSN) are used in different environmental applications such as, nuclear facility monitoring, forest fire detection, earthquake detection etc. and sending the sensed data to the database through a network. Today's sensor nodes are self configured and bi-directional. Yet, the wireless sensor node has resource constraints like low processing power, memory size and limited battery energy. Consequently, efficient energy utilization is an important issue in sensor networks.

WSN monitoring schemes have the advantage of easy maintenance, installation and cost effective. Exclusive of their updated technology, they flexible to storage of information and inspecting data when compared to traditional wired monitoring system. WSN based systems are now widely used for real-time environmental monitoring as well as in agriculture and aquaculture. They allow conditions to be monitored continuously on remote basis. This was proven for monitoring shortterm variations in these parameters, particularly in aqua ponds.

We propose to use Wireless sensor networks to have a distributed collection of sensor nodes ( $\mathrm{pH}$, Humidity, Temperature sensors etc.) networked together in some fashion so that they send the raw or processed sensed data to some central location called base station or database server through GSM. Every sensor node will have a microcontroller, some sensors and a radio transceiver for communication. This microcontroller can be used for in-network processing, so that only the necessary information can be transmitted instead of plain raw data. As the sensor nodes are battery-powered, any reduction in the number of bits transmitted will save power expensed on transmission, thereby extending battery life. There is a lot of research activity in this area addressing problems like sensor node placement for optimal coverage, topology formation, routing, in-network data processing techniques to reduce communication costs, operating system design etc. Most of this work is simulation based and the real life deployments are just a hand full, like Princeton's Zebra Net [4] and UCLA's Geo Net. There are few efforts in applying sensor networks for precision aquaculture [5]. It is hard to estimate their impact from these references, as we believe it will take few crop cycles to understand the on-field issues and refine the designs; and few more crop cycles to gather enough data for impact analysis. After analyzing the data in database we can send the data to the mobile phones of farmers in their local language to make them alert in unhygienic environmental conditions. With this proposed system even semi-literate farmers can interact with the system and understand the information generated to take suitable actions or precautions. The research issues are given below.

\section{Sensor networks in aquaculture: cost-effectively and utility studies}

We propose to deploy a sensor network using sensor nodes at the lowest cost possible in Aquaculture fields. The purpose of this is to understand the technical and on-field issues involved in the deployment of sensor networks and to perform a cost-benefit analysis.

\section{Design of low cost sensor nodes}

The cost of deploying a sensor network either in agriculture or in aquaculture using currently available off-the shelf sensor nodes is prohibitively expensive. Low cost sensor nodes have to be designed and developed to make a sensor network deployment economically feasible. We propose to explore the following approaches to reduce the sensor node cost.

1. We can possibly get away with the microcontroller and the radio transceiver in a sensor node by coming up with a design wherein the sensed parameters are encoded directly into a GSM modem. Data can be read from transmitter through wirelessly.

2. We can reduce radio transceiver costs by devising new short range communication protocols as against using either Bluetooth or ZigBee protocols which are widely popular in sensor networks.

The aforementioned approaches are feasible given that the sensor nodes need not form a self-organizing network as the terrain is very well accessible to humans in the case of aquaculture. However we have to explore the potential problems of such a design if automation for irrigation has to be done. 


\section{Data gathering, analysis and modeling}

Sensed parameters have to be gathered power efficiently and stored in a central database. Power efficient data collection strategies have to be investigated. Data mulling is a possible approach we can adopt with a hand-held cell phone acting as a mobile base station. Due to continuous collection of parameters, large amounts of data with spatial and temporal attributes will be generated. This data has to be stored in such a way that we can apply time-series data mining algorithms to extract patterns. We can use this data to train and build machine learning models to predict for example the possibility of a disease breakout. Similarly machine learning models could be built to predict crop yields.

\section{Mobile phone user interface design}

Farmers should be provided with an easy to use interface to allow them to access the information with temporal and spatial properties through appropriate visualizations and messages in local languages. For successful deployment and usage of the wireless sensor network technology in aquaculture, right user interface design for farmers could make the difference.

\section{Motivation}

In Aquaculture, the yields (shrimp, fish etc.) depend on the water characteristics of the aquaculture pond. For maximizing fish yields, the parameters which are to be kept at certain optimal levels in water are dissolved oxygen, temperature, salinity, turbidity, $\mathrm{pH}$ level, alkalinity and hardness, ammonia and nutrient levels. These parameters can vary a lot during the period of a day and can rapidly change depending on the external environmental conditions (Figure 1).

In early years, considerable amount of work has been carried out with respect to related issues yet communications with the outside world are still not yet completely investigated. Till now they have not yet ended up boundless and utilized within the different applications in which they can give huge profits. What's more, there are still just a set number of business WSN-based results accessible in the business. This is not amazing given the difficulties identified with their configuration and execution, power utilization demands of the sensor nodes, communication failures, environmental influence, and scalability issues. The distinguishment of these difficulties inspired us to perform this exploration. Specifically, this exploration proposes a model answer for the issues said above.

"Lin [10] created a Traceability enciphers for Recirculation Aquaculture (RATS) based on WSN. The figure empowers fast arrangement and can procure water temperature, saltiness, broke down oxygen and $\mathrm{pH}$, accomplish continuous Figures transmission. The RATS was mostly created utilizing C\# as a part of Microsoft Visual

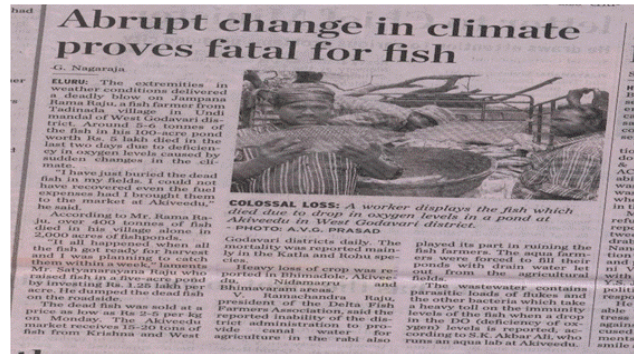

Figure 1: Paper cutting for problems in aquaculture.

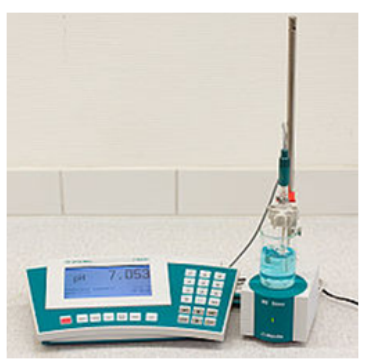

Figure 2: Manual testing of $\mathrm{pH}$.

Studio 2008 incorporated with the constant screen outline controlled by the Matlab M-dialect element join library [11].

"Created an online water checking framework focused around ZigBee and GPRS, the sensor information was gathered and transmitted by means of ZigBee and GPRS. The Data Process Procedure was actualized by lab view programming [12].

"Created an appropriated estimation framework focused around organized brilliant sensors to screen aquaculture considers in nature's turf. The framework comprises of four sections: information gathering hubs, steering hubs, on location observing focus and undemonstrative checking focus; and can bring-out ongoing observing water quality parameters and meteorological parameters [13]".

"Create a Zig-bee remote sensor system for observing an exploratory aquaculture re-circulating framework. Temperature, disintegrated oxygen, water and pneumatic force and also electric ebb and flow sensors were incorporated in the setup. The modules were introduced in an aquaculture re-circulating framework to transmit sensor qualities to the system facilitator. An observing system was created utilizing the C\# programming dialect to run on a windows PC with a specific end goal to show and store sensor values and to contrast them and reference limits. A remote framework for water quality web checking in escalated fish society, which joined web-server-inserted engineering with portable telecom innovation, was created by Zhu [14]. This framework was intended to conjecture water quality with simulated neural systems (Ann's) focused around recorded information and control the water quality in time to decrease cataclysmic misfortunes. At the same time, the creators of this work conceded that the application of their proposed framework is still constrained by its fairly convoluted operational prerequisites and high upkeep cost since it uses code division various access (CDMA) innovation for remote information transmission which obliges installment administration of sending information through portable system administrators [14].

Figure 2 Shown given below, In Existing system, farmer checks their ponds daily. And manually we will measure the $\mathrm{pH}$ value of water. $\mathrm{pH}$ is measured by the $\mathrm{pH}$ meter made of electronic device. Electronic meter is connected to $\mathrm{pH}$ probe (a glass electrode). It displays the readings.

The key part of $\mathrm{pH}$ meter is the probe. It is like a pole structure generally manfactured by the glass that contain a bulb at the bottom and also contain sensitive sensor. Suppose we want to find out solution, probe is dipped into the solution to easily get the readings.

Suppose the farmers want to find out the characteristics of water then they must collect water from the ponds and take to laboratory. Check the parameters of water samples. If those samples are not good results then they must take necessary actions. So this procedure 
takes a lot of time and manual testing is not possible in unhygienic environmental conditions

\section{Disadvantages of existing system:}

1. All $\mathrm{pH}$ meters need to be calibrated at some frequency

2. The probe has to be cleaned well after every use to make sure you get a reliable reading next time

3. It takes lot of time to test $\mathrm{pH}$ in manually

4. Manually testing is not possible in unhygienic environmental conditions

Some systems were developed with wireless sensor networks but some problems exist in the system, such as battery problem, communication protocols problems, sensor readings reliability. However, earlier systems were not made with GSM Network in aqua monitoring systems for remotely connecting environmental parameters such as $\mathrm{pH}$, humidity, inside temperature and outside temperature of water.

\section{Proposed System Description}

We propose the automatic system for checking water characteristics of aqua ponds in any situation (hygienic and unhygienic environmental conditions) (Figure 3). The system consists of two modules which are transmitter station and receiver station with personal computer as an analyzing center or monitoring center. The following list contributes overall framework of the aqua monitoring system.

\section{Embedded Systems Design}

2. Radio Frequency Integrated Circuits

3. Wireless Sensor Networks

4. The collected data will be analyzed by using Data Mining Tool

5. Convert the output into local language using local language translators

6. User interface design in local languages

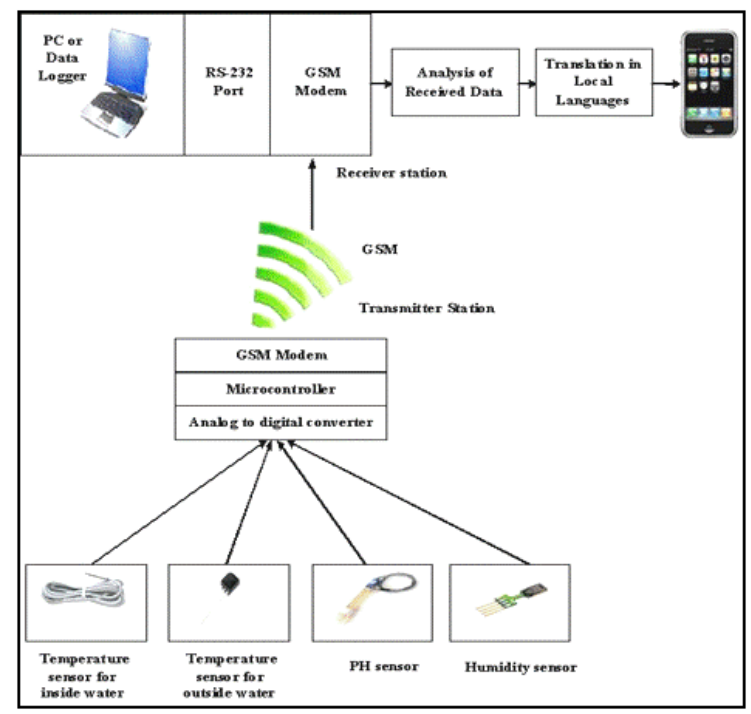

Figure 3: Overall architecture of aqua monitoring system.

\section{Design of transmitter station}

Wireless sensor networks have a distributed collection of sensor nodes (Temperature, $\mathrm{pH}$ and Humidity sensors) networked together in some fashion so that they transmit the raw or processed sensed data to some central location called base station. Each sensor node has a microcontroller, sensors and a radio transceiver for communication. The microcontroller can be used for in-network processing, so that only the necessary information can be transmitted instead of plain raw data. As the sensor nodes are battery powered, any reduction in the number of bits transmitted will save power expended on transmission, thereby extending battery life. There is a lot of research activity in this area addressing problems like sensor node placement for optimal coverage, topology formation, routing, in-network data processing techniques to reduce communication costs, operating system design etc. Most of this work is simulation based and the real life deployments are just a hand full, like Princeton's Zebra Net and UCLA's Geo Net. There are few efforts in applying sensor networks for precision aquaculture. It is hard to estimate their impact from these references, as we believe it will take few crop cycles to understand the on-field issues and refine the designs; and few more crop cycles to gather enough data for impact analysis. However to the best of our knowledge the sensor networks deployed in these systems use off-the-shelf available sensor nodes and no specific efforts are made in the hardware design cost reduction.

Figure 3 Shown below, we propose to use sensors in transmitter station to monitor aqua farms for relevant parameters such as $\mathrm{pH}$ levels, ambient humidity dissolved oxygen levels, temperature inside and outside water, ammonia levels etc. In contrast to other studies, the graphical user interface was designed in VB and .Net, so that farmers and investigators can observe, investigate and analyze the related data. The transmitter consists of:

1. Sensor nodes

2. GSM Modems

3. Microcontroller

4. ARM Processor

\section{Sensor nodes}

Sensors are electronic devices that measures response to vary in physical environmental conditions like humidity, sound, temperature, pressure etc. It senses signals in voltages $($ eg. $+5 \mathrm{v},-5 \mathrm{v})$ and continuously sends signal in the form analog signals and also converted into digital form by using an analog-to-digital converter and sent to microcontroller for further processing. They are in small size, consume low power, and operate in high densities.

Here we are using sensors

1. Temperature sensors for measuring temperature inside and outside water.

2. $\mathrm{pH}$ sensor

3. Humidity Sensor

\section{Sensor selection}

1. Water outside temperature sensor: This is the latest DS18B20 1-Wire digital temperature sensor from Maxim IC. Reports oc with 9 to 12-bit precision, $-55 \mathrm{C}$ to $125 \mathrm{C}( \pm 0.5 \mathrm{C})$. Each sensor has a unique 64 Bit Serial number etched into it, allowing for a huge number of sensors to be used on one data bus. This is a wonderful part that is the corner 
stone of many data-logging and temperature control projects. Unique 1-Wire interface requires only one port pin for communication. Each device has a unique 64-bit serial code stored in an onboard ROM. Multi drop capability simplifies distributed temperature sensing applications, requiring no external component to be powered from data line. Power supply range is $3.0 \mathrm{~V}$ to $5.5 \mathrm{~V}$. Measured temperatures are from $-55^{\circ} \mathrm{C}$ to $+125^{\circ} \mathrm{C}\left(-67^{\circ} \mathrm{F}\right.$ to $\left.+257^{\circ} \mathrm{F}\right) \pm 0.5^{\circ} \mathrm{C}$ accuracy and from $-10^{\circ} \mathrm{C}$ to $+85^{\circ} \mathrm{C}$. Thermometer resolution is user-selectable from 9 to 12 bits converting temperature to 12-bit digital word in $750 \mathrm{~ms}$ (max.). User-definable non-volatile (NV) alarm settings Alarm search command identifies and addresses devices whose temperature is outside of programmed limits (temperature alarm condition). Applications include thermostatic controls, industrial systems, consumer products, thermometers, or any thermally sensitive system. Electrical connections have to be done after any mechanical mounting. At the top of the multiple spot temperature sensors the lead out wires has to be connected either to the Tank Radar Rex gauge, the DAU, or a junction box [www.warf.com].

2. Water Inside temperature sensor: A temperature sensor is used with HOBO U-Series, UX120-006M external-channel data loggers or ZW series wireless data nodes. This model measures temperature in air, water, or soil. Accuracy and resolution vary according with attached logger model. Measurement range is $-40^{\circ}$ to $50^{\circ} \mathrm{C}\left(-40^{\circ}\right.$ to $\left.122^{\circ} \mathrm{F}\right)$ in water or soil, $-40^{\circ}$ to $100^{\circ} \mathrm{C}\left(-40^{\circ}\right.$ to $\left.212^{\circ} \mathrm{F}\right)$ in air; response time is minutes typical to $90 \%$ in air moving $1 \mathrm{~m} / \mathrm{sec}(2.2 \mathrm{mpH})$; response time in stirred water is 1 minute typical to $90 \%$. This model sensor is attached to a $1.8 \mathrm{~m}(6 \mathrm{ft})$ cable can be plugged directly into the external input jacks of U12, UX120, or ZW Family loggers to expand the range of measurement options and applications. Environment is the TMC6-HD Sensor is for use in Indoor, Outdoor and Underwater environments. Measurement Range is $-40^{\circ}$ to $50^{\circ} \mathrm{C}\left(-40^{\circ}\right.$ to $\left.122^{\circ} \mathrm{F}\right)$ in water; $-40^{\circ}$ to $100^{\circ} \mathrm{C}\left(-40^{\circ}\right.$ to $\left.212^{\circ} \mathrm{F}\right)$ in air ,Accuracy is w/U12: $\pm 0.25^{\circ} \mathrm{C}$ from $0^{\circ}$ to $50^{\circ} \mathrm{C}\left( \pm 0.45^{\circ} \mathrm{F}\right.$ from $32^{\circ}$ to $\left.122^{\circ} \mathrm{F}\right)$, insert probe $2.3 \mathrm{~cm}(0.9$ inches) minimum; see Plot A w/ZW: $\pm 0.21^{\circ} \mathrm{C}$ from $0^{\circ}$ to $50^{\circ} \mathrm{C}\left( \pm 0.38^{\circ} \mathrm{F}\right.$ from $32^{\circ}$ to $\left.122^{\circ} \mathrm{F}\right)$, insert probe $2.3 \mathrm{~cm}$ ( 0.9 inches) minimum; see Plot B w/UX120-006M: $\pm 0.15^{\circ} \mathrm{C}$ from $0^{\circ}$ to $70^{\circ} \mathrm{C}\left( \pm 0.27^{\circ} \mathrm{F}\right.$ from $32^{\circ}$ to $\left.158^{\circ} \mathrm{F}\right)$, insert probe $2.3 \mathrm{~cm}$ ( 0.9 inches) minimum; see Plots $\mathrm{C}$ and $\mathrm{D}$, Resolution is $\mathrm{w} / \mathrm{U} 12: 0.03^{\circ}$ at $20^{\circ} \mathrm{C}\left(0.05^{\circ}\right.$ at $\left.68^{\circ} \mathrm{F}\right)$; see Plot $\mathrm{A} \mathrm{w} /$ $\mathrm{ZW}: 0.02^{\circ} \mathrm{C}$ at $25^{\circ} \mathrm{C}\left(0.04^{\circ} \mathrm{F}\right.$ at $\left.77^{\circ} \mathrm{F}\right)$; see Plot B w/UX120-006M: $0.002^{\circ}$ at $25^{\circ} \mathrm{C}\left(0.003^{\circ}\right.$ at $\left.77^{\circ} \mathrm{F}\right)$; see Plots $\mathrm{C}$ and $\mathrm{D}$, Drift is $<0.1^{\circ} \mathrm{C}\left(<0.2^{\circ} \mathrm{F}\right)$ per year, Response time in air is $2 \mathrm{~min}$. typical to $90 \%$ in air moving $1 \mathrm{~m} / \mathrm{sec}(2.2 \mathrm{mph})$, Response time in stirred water is $30 \mathrm{sec}$. typical to $90 \%$.Operating range is Sensor tip and cable immersion in fresh water up to $+50^{\circ} \mathrm{C}\left(122^{\circ} \mathrm{F}\right)$ for 1 year, Housing is Copper-plated sensor tip ,Dimensions are $5.1 \times 33 \mathrm{~mm}(0.2 \times 1.3$ inches $)$ and Weight is $34 \mathrm{~g}(1.1$ oz) [15].

3. pH sensor: The most common $\mathrm{pH}$ sensor is the glass electrode. It is used in many industry applications and in a wide variety of fields. The glass-electrode method has high reproducibility, and it can measure $\mathrm{pH}$ of various solutions. A pH electrode is a potential metric or electrochemical sensor that has a voltage output. A potential metric sensor consists of two electrochemical cells or electrodes- The glass electrode, sometimes called the measuring electrode or active electrode, and the reference electrode. The electric potential created between the glass electrode, and the reference electrode is a function of the $\mathrm{pH}$ value of the measured solution. PI10 developed by Micro set instrumentation \& control $\mathrm{pH}$ probe is produced for the laboratory and industry use Sensitive stable long life, Adoption of the most advanced Teflon diap Hragm with large ring section make the electrode with stable liquid junction. Quick response with high sensitivity, the measured value of $95 \%$ can be obtained in a few seconds. The reference system is filled with high polymer; convenient to use and it is unnecessary to refill KCL solution, $\mathrm{pH}$ range is $0-14 \mathrm{pH}$, temperature range is $0-110$, pressure resistance: $0.6 \mathrm{MP}$ and zero potential $\mathrm{E}_{0}=7 \mathrm{pH}$, the external dimensions of the electrode is $12 \times 120,150,225 \mathrm{~mm}$ or other length. Unique structure preventing the electrode from easy pollution and blockage. The electrode is suitable for various media with severe pollution and it can prevent the spread of $\mathrm{S}^{2-}, \mathrm{NH}^{4+}$ and $\mathrm{CN}^{-}$, Plug-in connector; convenient for the installation and replacement of electrode, It can be installed with the thread PG13.5 [16].

Humidity sensor: Humidity is one of the most frequently measured quantities in different fields, such as industrial processing, agricultural, climate research, pharmaceutical engineering, etc. A humidity sensor is a device used to measure the humidity of air or any gas in a given area. It can be used in both indoors and outdoors. The humidity sensor is able to assists people's daily life in an invisible way. For example, to prevent the paper jam problem, the inside humidity sensor would detect the relative humidity at first, then the microchip would determine whether to warm up the printer to reduce the water vapor. The amount of water vapor in the atmosphere determines humidity. The humidity plays an important role in the numerous measurement situations. Hygrometry is in fact area branch of applied physics in which the multitude of techniques is an indication of the complexity of the problem, and of the fact that no one solution will meet all requirements at all times and in all places. Nowadays, there are many different types of humidity sensor to be set in various fields [Z.M.Rittersma]. HIH-4000-001 Humidity Sensor is $2.54 \mathrm{~mm}$ SIP, Sensing Accuracy is $3.5 \%$, Humidity Range is $0 \%$ to $100 \%$, Sensitivity is $\pm 3 \%$, Sensor Output 1 is Voltage, Sensor Terminals is Through Hole, Response Time is $15 \mathrm{~s}$, Supply Voltage Min: 4VDC, Supply Voltage Max is 5.8VDC, Operating Temperature Min is $-40^{\circ} \mathrm{C}$, Operating Temperature Max is $85^{\circ} \mathrm{C}$,SVHC: No SVHC (16Dec-2013), External Depth is $2.03 \mathrm{~mm}$, External Length/Height is 8.59 $\mathrm{mm}$, External Width is $4.17 \mathrm{~mm}$, Hysteresis is 3\%, Lead Spacing is 2.54 $\mathrm{mm}$, No. of Pins: 3 Operating Temperature Range is $-40^{\circ} \mathrm{C}$ to $+85^{\circ} \mathrm{C}$, Repeatability is $0.5 \%$, Sensor Supply Voltage is $4 \mathrm{~V} \mathrm{DC}$ to $5.8 \mathrm{~V} \mathrm{DC}$ and Supply Current is $0.5 \mathrm{~mA}$ [17].

\section{GSM modems}

Aquacultures ponds are far away to villages and power-supply not available to the ponds, data collection nodes are not placed at place. We are setup the receiver node at data collection center in villages or towns and also send the warnings or message to the farmers in unhygienic environmental conditions. So In this prototype we used the GSM modems for covers long range communication. gsm modem have a SIM card, operates under a mobile operator, just like a mobile phone. It support the $2 \mathrm{G}, 3 \mathrm{G}$ technologies, HSUPA, UMTS, WCDMA and HSDPA, as well as the GPRS and EDGE.GSM modems are embedded at both stations (transmitter and receiver station).

\section{Microcontroller}

In this prototype we used The AT89C52 is microcontroller with a low-power; high-performance CMOS 8-bit microcomputer with $8 \mathrm{~K}$ bytes of Flash programmable and erasable read only memory (PEROM). The device is manufactured using Atmel's high-density nonvolatile memory technology and is compatible with the industrystandard 80C51 and 80C52 instruction set and pin out. The on-chip Flash allows the program memory to be reprogrammed in-system or by a conventional nonvolatile memory programmer. By combining a versatile 8-bit CPU with Flash on a monolithic chip, the Atmel AT89C52 is a powerful microcomputer which provides a highly-flexible and cost- 
effective solution to many embedded control applications.

The AT89C52 provides the following standard features: $8 \mathrm{~K}$ bytes of Flash, 256 bytes of RAM, 32 I/O lines, three 16-bit timer/counters, a six-vector two-level interrupt architecture, a full-duplex serial port, on-chip oscillator, and clock circuitry. In addition, the AT89C52 is designed with static logic for operation down to zero frequency and supports two software selectable power saving modes. The Idle Mode stops the CPU while allowing the RAM, timer/counters, serial port, and interrupt system to continue functioning. This controller integrate and control the all the sensors.

\section{ARM processor}

In this prototype we used the ARM Processor, it is the industry's leading supplier of microprocessor technology, offering the widest range of microprocessor cores to address the performance, power and cost requirements for almost all application markets. Combining a vibrant ecosystem with over 1000 partners delivering silicon, development tools and software, more than 30 billion processors sold with more than 16M. ARM Holdings develops the instruction set and architecture for ARM-based products, but does not manufacture products. The company periodically releases updates to its cores. Current cores from ARM Holdings support a 32-bit address space and 32-bit arithmetic; the ARMv 8-A architecture, announced in October 2011,s support for a 64-bit address space and 64-bit arithmetic. Instructions for ARM Holdings' cores have 32 bits wide fixed-length instructions, but later versions of the architecture also support a variable-length instruction set that provides both 32 and 16 bits wide instructions for improved code density. Some cores can also provide hardware execution of Java byte codes.

ARM Specialist Processors are designed to meet the demanding needs of specific markets. Secure Core processors are utilized within the security markets for mobile SIMs and identification applications and integrate numerous technologies to detect and avoid security attacks while delivering outstanding performance. ARM also develops processors for FPGA fabrics, enabling users to rapidly reach market while maintaining compatibility with traditional ARM devices. Additionally the fabric independent nature of these processors enables developers to choose the target device which is right for their application rather than be locked to a specific vendor.

\section{System Software}

The aqua monitoring system checking with various sensors records also saves the entire measured data to make an aquaculture database system, which provides analysis of date by using analyzed patterns of changing environmental conditions in the fish ponds. That Information compared with preset ideal conditions. If a measure data from a monitoring system is outside a preset range, the system sends warning to the farmers to take suitable actions. Communication process module connected to the PC via serial port RS-232.

\section{Sensor node firmware}

The embedded software to control the wireless sensor system is actualized utilizing IAR or RAM created in C language. The hex document is then ported to the flash memory of the ATmega8 microcontroller via USB interface. The project is created with subroutine modules for sensor indicator digitization, engine transfer and valve hand-off control, remote information procurement and transmission, and universal and asynchronous receive and transmit (UART). Analog to digital converter (ADC) is used for converting signals from analog format to digital format. The microcontroller ADC performs cyclic scans of those sensors. The check rate is programmable and can be adaptive based on the rate of vary of sensor readings.

The sensors information might be contrasted and set-point qualities put away in memory. Serial correspondence at 9600 baud rate is carried out and information is given to GSM transceiver which transmits information to collector by means of GSM remote system. The recipient station receives the information and goes to $\mathrm{PC}$ at baud rate of $9600 \mathrm{bps}$ and Crystal_frequency_in_hz 12000000 serial interface. Information obtaining and transformation of crude information is carried out by utilizing ARM processor utilizing IAR 5.4_2 KICK (Figure 4).

\section{GSM network communication software}

In this paper we implemented the GSM communication protocol for sending the data from transmitter to the receiver. It covers long range and directly send the data to databases or Farmers mobile Phones. The GSM (Table 1) modems are placed at both sides of transmitter and receiver. The Transmitter needs to start working first. After power is switched on, it initializes the protocol stack and then performs energy detection; it selects the appropriate channel and starts the GSM modem. Then sensor nodes can be connected, accepted and the transmission of sensor data from each node to PC is done. Transmitter process flow diagram is shown in Figure 5.

After power is switched on, the sensor nodes does the first scan, looks for a transmitter and then establishes a connection with microcontroller. If the connection is successful, it synchronizes through the microcontroller to the transmitter, and collects the parameter by collection cycle, and finally sends the measurements to the receiver station. Receiver process flow diagram is shown in Figure 6.

\section{Monitoring interface software}

For this project IAR 5.4_2 KICK software development environment has been chosen. It gives powerful comprehensive group of software tools and functional libraries for analysis, presentation and data acquisition. To develop data acquisition, analysis and presentation to interactively IAR 5.4_2 KICK flexibility to provides all these libraries

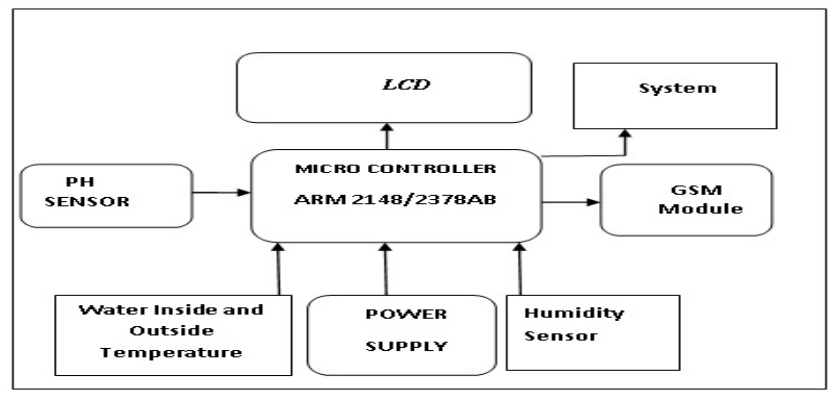

Figure 4: Block diagram of transmitter.

\begin{tabular}{|l|c|c|c|c|}
\hline Parameters & ZigBee & Bluetooth & Wi-Fi & GSM \\
\hline Range & $30 \mathrm{~m}-1.6 \mathrm{KM}$ & $30-3000 \mathrm{ft}$ & $100-150 \mathrm{ft}$ & $30-35 \mathrm{KM}$ \\
\hline $\begin{array}{l}\text { Power } \\
\text { Consumption }\end{array}$ & Low & Medium & High & High \\
\hline Frequency & $2.4 \mathrm{G}$ & $2.4 \mathrm{G}$ & $2.4 \mathrm{G}$ & 900 and $1800 \mathrm{MHz}$ \\
\hline $\begin{array}{l}\text { Protocol/ } \\
\text { Modulation }\end{array}$ & CSMACA, DSSS & FHSS & CCK, OFDM, DSSS & $\begin{array}{c}\text { MS, BTS, } \\
\text { BSC,MSC }\end{array}$ \\
\hline Cost & Low & Low & High & Medium \\
\hline \multicolumn{4}{|r|}{ Table 1: Technical specifications of communication protocols. }
\end{tabular}




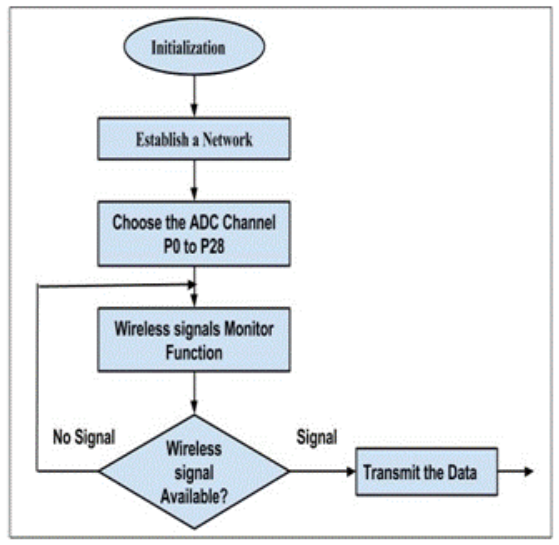

Figure 5: Transmitter processes flow diagram.

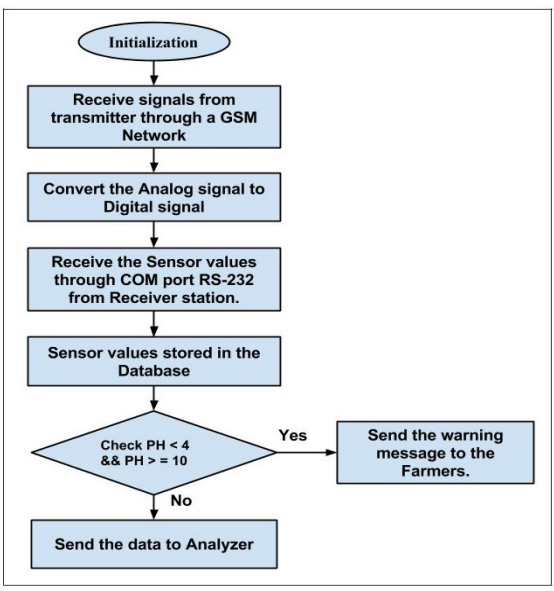

Figure 6: Receiver processes flow diagram

in $\mathrm{C}$ language and easy to use tools for design of aqua monitoring system. It consists driver software's, flash magic libraries, Analog to digital converter programs, sensor node programming, database connectivity programs.

However, IAR 5.4_2 KICK provides scalability, modularity and customization than conventional software. We can edit, compile, debug and link with C programs in the IAR 5.4_2 KICK development environment. On the other hand compiled $\mathrm{C}$ object files, dynamic linking libraries, drivers, $\mathrm{C}$ libraries connected to the $\mathrm{C}$ source file.

Aqua monitoring system have a three steps, they are data acquisition, analyzing and presentation. Hardware device gives sensing data to the data acquisition system, analyzing by the data mining tools and presentation developed in VB OR dot NET. That Graphical user interface created control to hardware device for data acquisition and also display the data. Analysis is done by performing scaling, signal processing, formatting, statistical analysis, and curve fitting. Data displayed in the form of strip charts, graphs etc. pull down menus and pop-up dialog boxes are created for giving inputs to the graphics. The user interface elements designed interactively by the GUI editor and also configure and create programmatically. Data acquisition, data analysis and user interface control by the program control. it manages the flow of program execution. IAR 5.4_2 KICK takes advantage of multithreading which benefits data acquisition and instrumental control applications including improved responsiveness, throughput and more efficient background processing.

Task can be divided as multithreads. Sometimes when the lowest priority thread is executing, it is interrupted by the highest priority thread. Then the lowest priority thread goes into wait queue. Thread switches are performed by the operating system. The total waiting time is reduced through input/output operations are performed simultaneously. It improves the multiprocessor performance by making each processor to execute a thread. So, more than one task can be executed simultaneously. For example acquiring data, analyzing the data and present the analyzed data on the graphical user interface.

The controlling feature is added in the graphical user interface (GUI) designed in IAR 5.4_2 KICK to control various devices automatically and manually such as relays and water valves. In the automatic controlling of humidity and temperature, each device can start and stop recording to the range values set for them. While in manual controlling, one can start and stop any device according to the need by pressing the relay knob to the relevant position. The monitoring software consists of four subroutines. The communication subroutine is liable for proper configuration of the ports, the acquire data is send to the database and command signals for controlling output relays. It also sets protocols for communication with all devices in every node interconnected to the system.

It includes a $\mathrm{pH}$ monitor, water temperature monitor, and humidity monitor. In addition, it displays graphical and numerical values of all monitored data in real time. The archive management module is responsible for storing and maintaining previous 1 data in aquaculture. Sensor nodes gives data to the database for the store the fundamental data and generates reports in word spreadsheet, option for performing calibration of sensors, indicates the status of output relays and network status, and provides help option in daily production.

\section{System implementation}

The system (Figures 7 and 8) was implemented in Gudlavalleru, Krishna district. Intensive aquaculture monitoring system acquire the data from March-15 2014 to September-10, 2014. This system observes for the appropriate operation of the system and assesses the feasibility. The pond area is 3 acres, divided into four fish ponds. Four sensors nodes were deployed in fish pond. Water inside and outside temperature sensors, $\mathrm{pH}$ sensor and humidity sensors were installed as input devices at transmitter node. The system was tested connecting the sensors to the modules and the receiver node to the monitoring computer in the control room, installation of sensor nodes in the fish ponds and their operation.

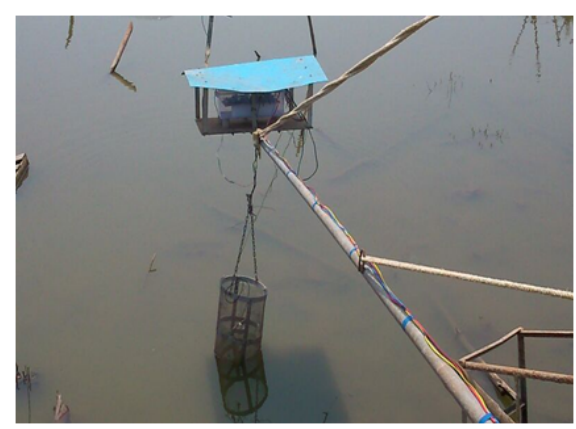

Figure 7: Installation of Transmitter station at pond. 
Citation: Chandanapalli SB, Sreenivasa Reddy E, Rajya Lakshmi D (2014) Design and Deployment of Aqua Monitoring System Using Wireless Sensor Networks and IAR-Kick. J Aquac Res Development 5: 283. doi:10.4172/2155-9546.1000283

Page 8 of 10

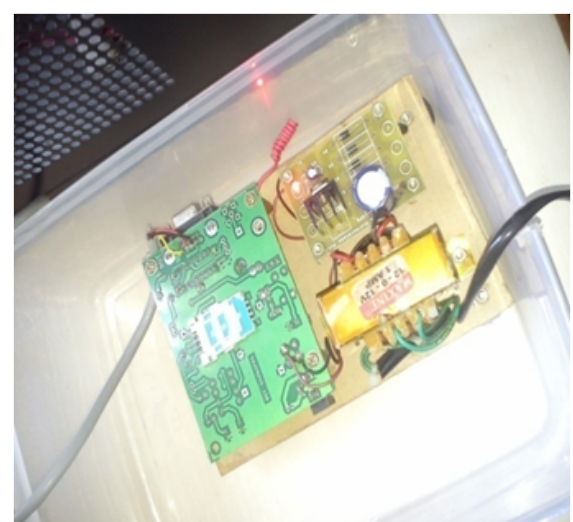

Figure 8: Receiver Station.

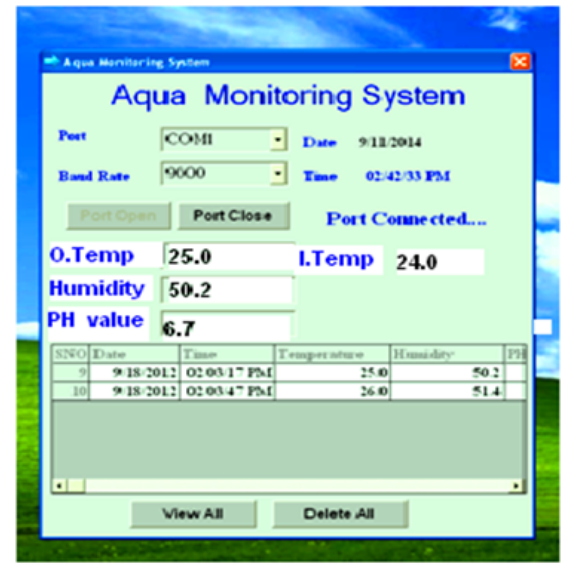

Figure 9a: Monitored data of Aqua monitoring System.

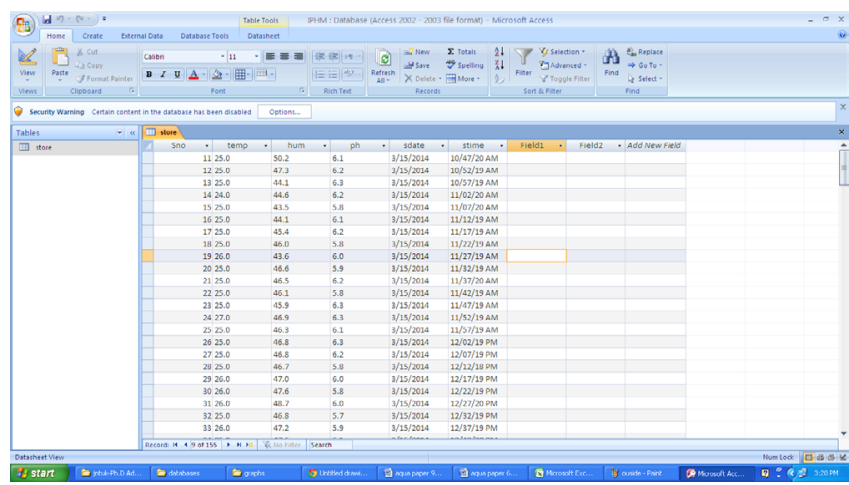

Figure 9b: Database of Aqua monitoring System

\section{Results and Discussion}

Storing data in the database (Figure 9b) of aquaculture environmental information sent from each sensor nodes in real time and also monitoring data show in Figure 9a. So that the user can have access to aquaculture environmental information. Information can be displayed as graph (Figures 10-15), or opened as datasheet with which user can understand the condition of aquaculture environment and take suitable action for any feasible problems in appropriate time. The database for storing sensor values consists of one table for all sensors where the index, date, time and sensor values are stored. The monitoring program can be configured to store data every selected time interval in seconds. The monitoring program displays an instrument panel with the latest values stored into the database and automatically updates to display the new values The measured data change consistently and reasonably reflecting the values from field sensors.

Figure 10 the graphical user interface was designed in VB and .NET, so that farmers and investigators can observe, investigate and analyze the related data. The user interface allows us to convey the analyzed data in the form of a message to the farmers in their respective local languages to their Mobile Phones and alerts them in unhygienic

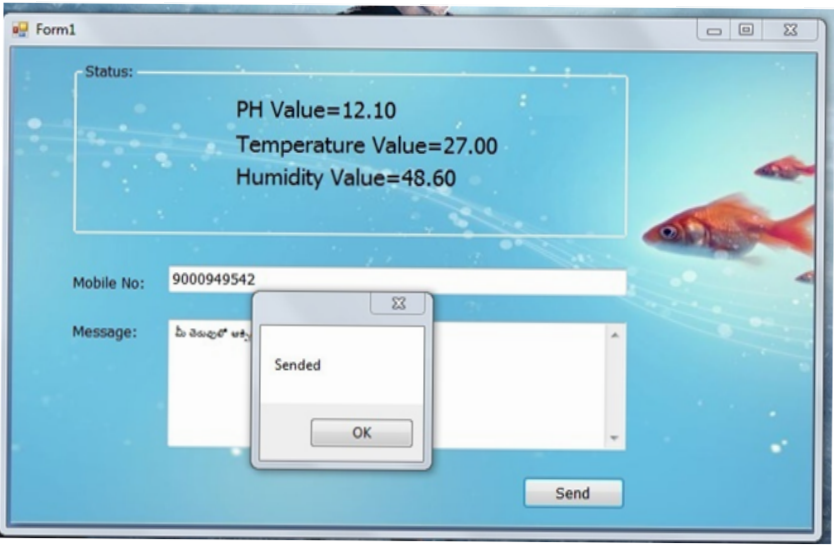

Figure 10: User interface of Aqua monitoring System.

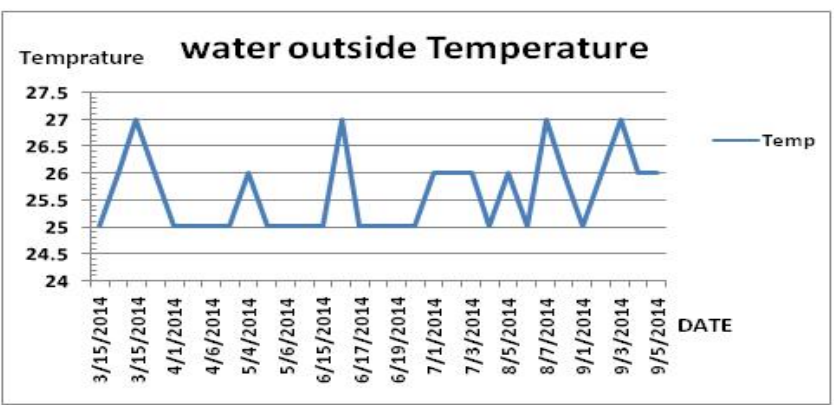

Figure 11: Water outside temperature variation from for a period of six months.

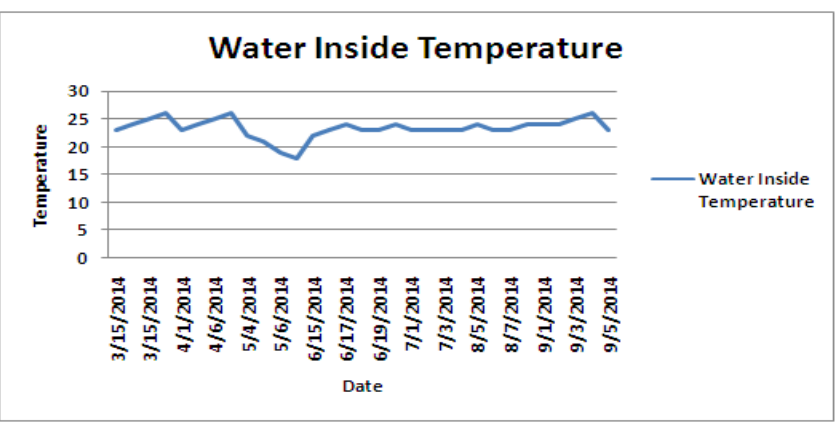

Figure 12: Water inside temperature variation from for a period of six months 


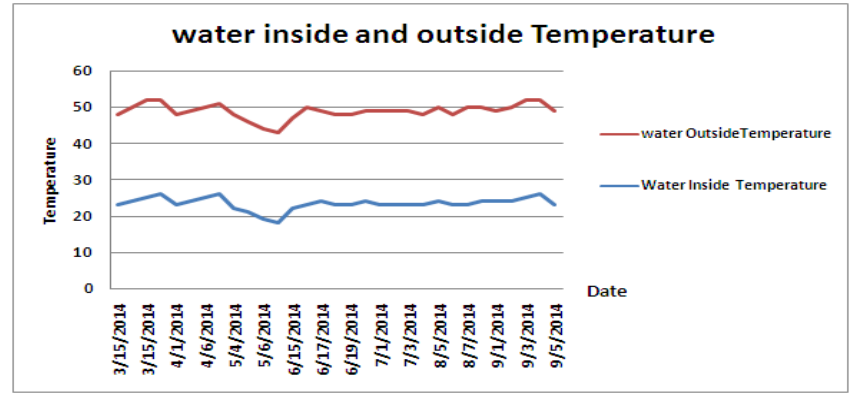

Figure 13: Water outside and inside temperature variation from for a period of six months.

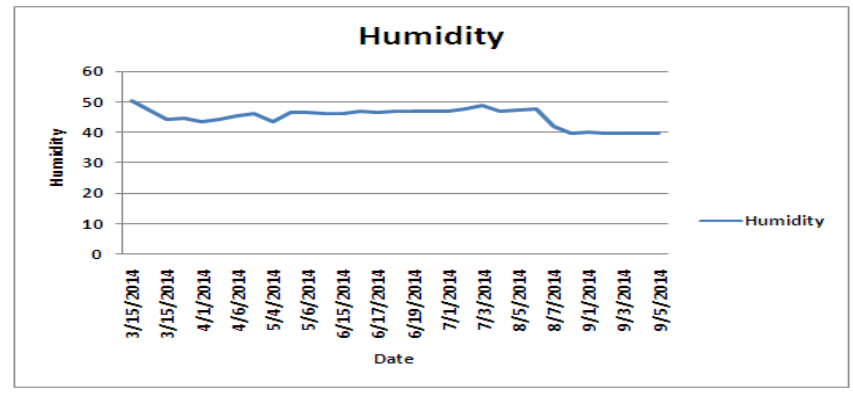

Figure 14: Humidity variation from for a period of six months.

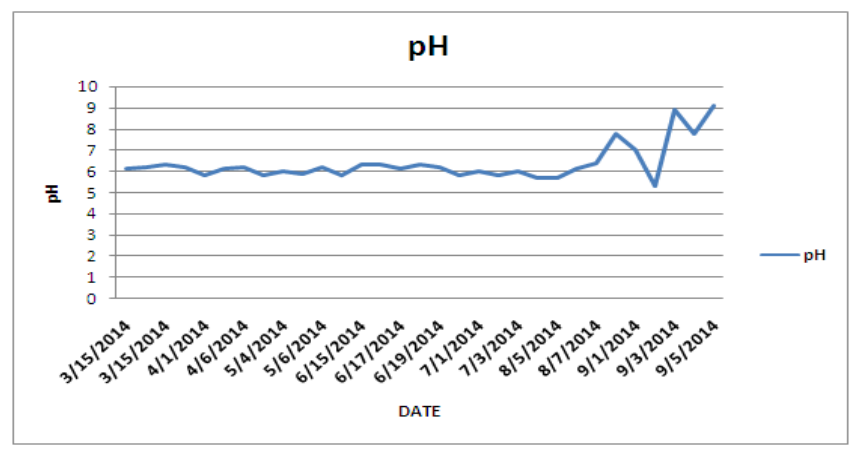

Figure 15: $\mathrm{pH}$ variation from for a period of six months.

environmental conditions. With this even semi-literate farmers can interact with the system and can understand the information in order to take suitable actions.

Figures 11-15 shows the data variation: (a) water outside temperature, (b) water inside temperature, (c) Temperature inside and outside of water (d) humidity (e) $\mathrm{pH}$, collected for a period of six months. The nodes were working properly providing some assurance of the robustness of the system.

Table 2 shows the statistical performance of our system for various parameters in six months. Fish mortality reduced significantly and environmental parameters were ascertained in real-time and as a result optimum condition suitable for fish growth and disease reduction were achieved. The standard deviation of all measurements is also shown. In our deployment, we used manual measurement of humidity, $\mathrm{pH}$ and water inside/outside temperature to verify the data. The results are not shown but were matching well.

Dissolved oxygen level was constant at night sustained by aerator pumps. However, during the day most of the time the aerator pumps was idle, due to enough humidity level depending on daily weather. In some cases especially on cloudy days, even during the day the ponds humidity level had to be sustained by aerator pumps. The relays were working properly at each node. Remote actuation of water valve and aerator pump was successfully operated by the wireless networks. That representing that the firmware and software were properly operated. They were automatically switching on/off whenever the parameters were outside preset range. The status light emitting diodes (LEDs) at the GUI were used to indicate the status of GSM communication, data transmission, relay states and terminal states. The latest parameters at each terminal were displayed in numerical and graphical by selecting the respective parameter of interest. The control dialog allows selecting of terminal number for performing data acquisition on and plotting graph in real-time. Additional functions of the system for performing sensor calibration, data saving, report generation and help options were functioning properly. It also allows manual starting and stopping the relays by pressing the relay knobs in respective positions.

Communication range depends on transmitter and receiver parameters, the output power of the transmitter and the sensitivity of the receiver and several external parameters. These parameters includes the physical environment particularly objects in or close to communication path which result in multipath propagation, signal scattering and attenuation as well as other interference sources. The communication range decreases as the quantity of tall buildings, trees and foliage near the fishponds increases. The presence of vegetation appears to impair communication by reducing the span of the connected area. Sometimes short outages occurred because of heavy rain and thunderstorms causing signal cutoff. When the system reset was done the signal got well operating normally.

Water inside and outside temperature, dissolved oxygen and $\mathrm{pH}$ were regularly monitored by this system. For example, water characteristics were maintained at best levels that is $\mathrm{pH}$ level is maintained between 4 to 10 . The smart sensor nodes are small, low cost, efficient and suitable for deployment in harsh environment. The nodes were protective watertight housing and weatherproof. Making it resistant to environmental factors like rain and heat. It was easy to move the nodes to the desired point of interest whenever needed. The experimental results reported in this work show that the power management and networking solutions were adopted to work in practice. This increased economic benefit for aquaculture by improving production process in quality and quantity, consumer confidence and safety. This system could prove to be helpful in the event of a failure to take rapid actions to prevent the damage that could be caused to the fish stock.

\section{Conclusion}

A wireless sensor network for aquaculture monitoring and control based on virtual instruments is presented. In addition, a detailed hardware design of the smart sensor nodes and transmitter/receiver with their software design is also presented. Finally, the prototype system with four smart sensor nodes and a transmitter/receiver is designed and implemented. The collected data provides an accurate analysis of successful operation of the system. This work can be used in various application areas such as greenhouse monitoring and control, hydrological water conservation and farmland irrigation. Future development efforts should involve enhancing the WSN 
Citation: Chandanapalli SB, Sreenivasa Reddy E, Rajya Lakshmi D (2014) Design and Deployment of Aqua Monitoring System Using Wireless Sensor Networks and IAR-Kick. J Aquac Res Development 5: 283. doi:10.4172/2155-9546.1000283

Page 10 of 10

\begin{tabular}{|c|c|c|c|c|c|c|c|c|c|c|c|c|}
\hline & \multicolumn{3}{|c|}{ Mar-14 } & \multicolumn{3}{|c|}{ Apr-14 } & \multicolumn{3}{|c|}{ May-14 } & \multicolumn{3}{|c|}{ Aug-14 } \\
\hline & Min & Max & Stdiv & Min & Max & Stdiv & Min & Max & Stdiv & Min & Max & Stdiv \\
\hline Water Inside Temperature & 23 & 26 & 2.12 & 23 & 25 & 1.40 & 18 & 22 & 2.83 & 22 & 24 & 1.41 \\
\hline Water Outside Temperature & 25 & 27 & 1.41 & 25 & 25 & 0.00 & 25 & 26 & 0.70 & 25 & 27 & 1.41 \\
\hline Humidity & 44.1 & 50.2 & 4.30 & 43.5 & 46 & 1.73 & 43.6 & 46.6 & 2.12 & 45.9 & 46.9 & 0.71 \\
\hline $\mathrm{pH}$ & 6.1 & 6.3 & 0.14 & 5.8 & 6.2 & 0.28 & 5.8 & 6.2 & 0.28 & 5.8 & 6.3 & 0.35 \\
\hline Fish Mortality & & 1.4 & & & 1.1 & & & 1.0 & & & 1.2 & \\
\hline \multicolumn{9}{|c|}{ June -14 } & \multicolumn{4}{|c|}{ Sep-14 } \\
\hline & Min & Max & Stdiv & Min & Max & Stdiv & Min & Max & Stdiv & & & \\
\hline Water Inside Temperature & 23 & 23 & 0.00 & 23 & 24 & 0.71 & 23 & 26 & 2.12 & & & \\
\hline Water Outside Temperature & 25 & 26 & 0.71 & 25 & 27 & 1.41 & 25 & 27 & 1.41 & & & \\
\hline Humidity & 46.8 & 48.7 & 1.34 & 42 & 47.6 & 3.96 & 39.7 & 39.9 & 0.14 & & & \\
\hline $\mathrm{pH}$ & 5.7 & 6 & 0.21 & 5.7 & 7.8 & 1.48 & 5.3 & 9.1 & 2.69 & & & \\
\hline Fish Mortality & & 1.0 & & & 1.3 & & & 1.5 & & & & \\
\hline
\end{tabular}

Table 2: Summary of sensor data between May-2014 to Sep-2014.

by adding GPRS modules on the smart sensor nodes in order to monitor aquaculture environment on-line through the internet. The monitoring program should be linked to the web server through a passive IP address in one port of the receiving computer, thus making the architecture more scalable and robust. This work also extended to agriculture monitoring.

\section{Acknowledgment}

This work was supported by ITRA project and also given Expression of Interest for Wireless Sensor Networks for Precision Agriculture and Aquaculture and is also sanctioned fund in in-house R\&D by Gudlavalleru Engineering College.

\section{References}

1. http://www.nmfs.noaa.gov/aquaculture/what_is_aquaculture.html

2. Ferreira N, Bonetti C, Seiffert W (2011) Hydrological and water quality indices as management tools in marine shrimp culture. Aquaculture 318: 425-433.

3. Harun A, Ndzi DL, Ramli MF, Shakaff AYM, Ahmad MN, et al. (2012) Signal Propagation In Aquaculture Environment For Wireless Sensor Network Applications. Prog Electromag Res 131: 477-494.

4. Glasgow, Howard B, Burkholder, JoAnn M, Reed, et al. (2004) Real-time remote monitoring of water quality: are view of current applications, and advancements in sensor, telemetry and computing technologies. J Exp Mar Biol Ecol 300: 409-448.

5. Zhuiykov S (2010) Water Quality: Physical, Chemical and Biological Characteristics. Nova Science, New York.

6. Bosma, Roel H, Verdegem, Marc CJ (2011) Sustainable aquaculture in ponds: principles, practices and limits. Livestock Sci 139: 58-68.
7. John Hargreaves A (2006) Photosynthetic suspended-growth systems in aquaculture. Aquacult Eng 34: 344-363.

8. Chien $Y$ (1992) Water quality requirements and managements for marine shrimp water culture. In: Proceedings of the Special Session on Shrimp Farming. World Aquaculture Society, USA.

9. Carbajal J, Sanchez L, Progrebnyak L (2011) Assessment and prediction of the water quality in shrimp culture using signal processing techniques. Aquacult. nt 19: 1083-1104.

10. Lin Qi, Jian Zhang, Mark Xu, Zetian Fu, Wei Chen, et al. (2011) Developing WSN-based traceability system for recirculation aquaculture. Math Comput Model 53: 2162-2172.

11. Wang Xin, Ma Longquan, Yang Huizhong (2011) UXI-LY water level sensor

12. Haijiang Tai, Shuangyin Liu, Daoliang Li, Qisheng Ding, Daokun Ma (2012) A multi-environmental factor monitoring system for aquiculture based on wireless sensor networks. Sensor Lett 10: 265-270.

13. Epinosa-Faller FJ, Rendon-Rodriguez GE (2012) A ZigBee wireless sensor network for monitoring an aquaculture recirculating system. J Appl Res Technol 10: $380-387$.

14. Zhu X, Li D, He D, Wang J, Ma D, et al. (2010) A remote wireless system for water quality online monitoring in intensive fish culture. Comput Electron Agric 715: 53-59.

15. http://www.onsetcomp.com.

16. http://www.all-about-pH.com.

17. www.topqualitytools.co.uk 